\title{
Editorial
}

\section{A renaissance in low-level laser (light) therapy - LLLT}

\author{
Renaissance der Low-Level-Laser(Licht)therapie - LLLT
}

This special issue on low-level laser (light) therapy (LLLT) has been assembled by soliciting individual contributions from a broad spectrum of specialists in the field throughout the world.

LLLT also known as photobiomodulation, is the drug-free, non-invasive and safe (FDA approved) clinical application of light - usually produced by low to mid power lasers or light emitting diodes (LED) with a power output in the range of $1-500 \mathrm{~mW}$ - to a patient to promote tissue regeneration and healing, reduce inflammation and relieve pain. The light is typically of narrow spectral range (1-40 $\mathrm{nm}$ bandwidth), in the visible (red) or near infrared (NIR) spectrum (600-1000 nm), and achieves an average power density (irradiance) between $1 \mathrm{~mW} / \mathrm{cm}^{2}$ and $5 \mathrm{~W} / \mathrm{cm}^{2}[1]$.

The reason why the technique is termed "lowlevel" or "cold laser" therapy is that the optimum level of energy density $\left(\mathrm{J} / \mathrm{cm}^{2}\right)$ delivered to the tissues is low when compared to other forms of laser therapy such as those used for ablation, cutting, and thermally coagulating tissue. In general, the power densities used for LLLT are lower than those needed to produce heating of tissue (i.e., $<100 \mathrm{~mW} / \mathrm{cm}^{2}$, depending on wavelength and tissue type), and the total energy delivered to the treated area is usually below $10 \mathrm{~J} / \mathrm{cm}^{2}$. A common reference is the "Guidelines for Skin Exposure to Laser Light" in the International Standards Manual (IEC-825) which considers an exposure below $200 \mathrm{~mW} / \mathrm{cm}^{2}$ as a safe exposure [2].

LLLT, i.e., the use of low levels of visible or NIR light for reduction of inflammation and pain [3], healing of wounds, muscle and nerve injuries [4], and prevention of tissue damage by reducing cellular apoptosis (for example, due to ischemia and reperfusion injury) [5], has been known for almost 50 years since shortly after the invention of lasers in 1960 [6]. However, despite many reports of positive findings from experiments conducted in vitro, in animal models and in randomized controlled clinical trials, the mechanisms of LLLT in biological tissues remains not fully elucidated. It is likely this is due to two main reasons: firstly, the complexity in interpretation of scientific and clinical data generated in different labs and clinical settings, and secondly, inconsistency in the use of light sources (medical devices) and treatment protocols including, illumination parameters (such as wavelength, fluence, power density, pulse structure, etc.) and the treatment schedule. Unfortunately, these variations led to an increase in the number of negative studies and created some controversy despite the overwhelming number of positive clinical results [7]. It is noteworthy that many preclinical and clinical studies have been and still are conducted without proper scientific methodology. Therefore, it is critical that all the characteristics of the light emitted by lasers or LEDs must be specified if data are to be useful. A requirement for a good phototherapy study, using LLLT in the visible or NIR region, whether from a laser, an LED, or a filtered incandescent lamp is to specify everything about the light source, i.e., wavelength(s), total power, power density, total energy, fluence, coherence, pulse structure (both frequency and pulse duration), polarization, area of exposure, illumination time, and treatment repetition [8]. There are published experimental and clinical studies that were conducted with good scientific methodology, but they did not fully describe the light parameters, therefore these studies cannot be repeated or extended by another researcher. Such publications are useless.

In recent years, major advances have been made in understanding the mechanisms that operate at the molecular, cellular and tissue levels during LLLT. Mitochondria are thought to be the main site for the initial effects of light. New discoveries in the last decade have significantly altered our view on mitochondria. They are no longer viewed as only energy-making cellular compartments but rather individual cells-within-the-cell that are involved in numerous signalling and second messenger pathways [9]. In particular, it has been suggested that many important cellular components such as specific enzymes and ion channels [for instance nitric oxide synthase (NOS), ATPdependent $\mathrm{K}^{+}$channels, and poly-(APD-ribose) polymerase, have a distinct, mitochondrial variant]. For example, the intriguing possibility that mitochondria are significant 
sources of nitric oxide (NO) via a unique mitochondrial NOS variant [10] has attracted intense interest among research groups because of the potential for NO to affect functioning of the electron transport chain [11]. One theory maintains that the fundamental molecular process in LLLT is the photodissociation of inhibitory NO from cytochrome c oxidase (unit IV in the respiratory chain) thus increasing ATP production, oxygen consumption, raising mitochondrial membrane potential and modulating production of reactive oxygen species [12]. However, other groups have proposed that cellular membranes, ion channels, porphyrins and flavoproteins may act as primary photoacceptors [13]. There is likely to be more than one reaction involved in the primary mechanisms of LLLT. There are no grounds to believe that only one of these processes occurs when tissue is irradiated. An important question for future research is which of these reactions is responsible for a certain wavelength effect.

One of the key factors that affect the efficacy of LLLT is the realization that there exists a biphasic dose response that directly correlates with the characteristics of the wavelength and the amount of energy absorbed by chromophore or photoacceptor, the light energy density (the radiant exposure or light fluence if measured below the surface) [14]. The important role of biphasic response has been demonstrated by a number of studies in cell culture, animal models, and in clinical studies [15].

Scientific evidence continues to demonstrate that pulsed light does have biological and clinical effects that are different from those of continuous wave (CW) light. Several studies revealed that the LLLT in pulsed wave mode of operation can better penetrate through the melanin and other skin barriers, supporting the hypotheses that pulsing is beneficial in reaching deep target tissue and organs [16].

The data undeniably demonstrate the benefit and the advantageous effect of pulsing in LLLT and are also providing a new insight into the molecular mechanisms associated with the LLLT clinical efficacy $[17,18]$. While CW and standard pulsed lasers are limited to $<1-2 \mathrm{~cm}$ therapeutic efficient penetration, superpulsed infrared laser technology is able to show therapeutic efficacy at up to $10 \mathrm{~cm}$ into tissue and benefit target tissues such as: bones, tendons, ligaments and cartilage. Many applications of LLLT may require deep penetration of the light energy, such as: lower back, neck, and hip joint pain and inflammation. Therefore, if the power densities needs to be greater than a few $\mathrm{mW} / \mathrm{cm}^{2}$ and is to be delivered safely to target tissues $>5 \mathrm{~cm}$ below the skin surface, the effective treatment can only be achieved by using superpulsed lasers [19]. The data indicate that pulsing and particularly superpulsing is playing an important role in LLLT especially for clinical applications where deep tissue penetration is required.

After a chequered history, the current renaissance in LLLT has been fueled in part by the realization of its immuno-regulatory properties [20], its neuroprotective properties [21] and its preferential action on stem cells and progenitor cells [22]. The spectrum of inflammatory mediators, upregulated in chronic pathological conditions, suggests many routes for future therapeutic intervention. It may be that multiple wavelengths administration, targeted at different inflammatory mechanisms, will prove far more effective than utilization of any single agent. In many cases, the data suggest exploiting targets that so far have not been considered important in the immunological field.

This interesting topic is addressed in a review by Hahm et al. [23] who discuss the role of the "pain, inflammation and immune response (PII) axis" in LLLT. These authors cover the role of inflammatory cytokines, neuropeptides, prostanoids, kinins, and histamine in pain and inflammation and how they may respond to LLLT.

A review by Vatansever and Hamblin [24] discusses the use of far-infrared radiation (FIR, $\lambda=3-12 \mu \mathrm{m}$ ) that has been shown to have biological effects that are similar to the more commonly employed red and NIR wavelengths. In addition to being delivered by light sources, these wavelengths can be produced by FIR-emitting ceramic nanoparticles that can be included into wearable garments that require no external power source.

A third review by Ferraresi et al. [25] concentrates on the effect of LLLT on muscle tissue. Due to the large number of mitochondria present in myocytes, muscles respond very well to LLLT. Treatment can be used both to increase healing, and decrease pain and inflammation after muscle injury and to decrease muscle fatigue and increase performance in athletes.

The first experimental paper is also on LLLT for muscle disorders. Vatansever et al. [26] report that LLLT delivered to aged rats after cryoinjury of the tibialis anterior muscle was able to increase expression of MyoD and VEGF genes, increase capillary density and improve maturation of satellite cells into myoblasts and myotubes.

Marquina et al. [27] carried out a randomized placebocontrolled clinical trial to evaluate a dual wavelength, multiple diode laser cluster probe with five super-pulsed 905 $\mathrm{nm}$ NIR laser diodes, each emitting at $40 \mathrm{~mW}$ average power and four CW $660 \mathrm{~nm}$ visible red laser diodes, each emitting at $25 \mathrm{~mW}$ in combination with standard chiropractic techniques on 126 patients with osteoarthritis and knee pain. Improvement was found in visual analog scale for pain relief 
and osteoarthritis measures, with a statistical and clinical significance of $p<0.01$ from baseline to the 30-day follow-up.

Almuslet and Osman [28] treated 24 patients with chronic plaque psoriasis with PDT using three CW diode lasers with red wavelengths $(671-675 \mathrm{~nm})$ with output powers of 16,50 and $100 \mathrm{~mW}$ ( 8 patients per group) to activate the photosensitizer (Levulan ${ }^{\circledast}$ Kerastick $^{\circledast}$ topical solution). Sixty-two percent of the patients treated with $100 \mathrm{~mW}$ achieved complete clearance compared with $25 \%$ for those treated with $50 \mathrm{~mW}$ and $0 \%$ for those treated with $16 \mathrm{~mW}$.

It is a great honor for us to act as guest editors for this special issue. We hope that the fast growing field of LLLT will continue to offer painless, non-invasive and highly effective drug-free solutions that will be recognized by every major industrialized nation in the world for mitigation of a plethora of medical conditions and will be widely available to the highly trained practitioner to control disease, particularly when conventional therapies have failed or have unacceptable side effects. Many thanks to all the authors for their excellent contributions.

Acknowledgements: Research in the Hamblin laboratory is funded by NIH grant \# RO1AI050875.

\section{References}

[1] Chung H, Dai T, Sharma SK, Huang YY, Carroll JD, Hamblin MR. The nuts and bolts of low-level laser (light) therapy. Ann Biomed Eng 2012;40(2):516-33.

[2] IEC 60825-1 - Ed. 2.0: 2007-03: Safety of laser products Part 1: Equipment classification and requirements. International Electrotechnical Commission, Geneva, Switzerland.

[3] Bjordal JM, Couppé C, Chow RT, Tunér J, Ljunggren EA. A systematic review of low level laser therapy with locationspecific doses for pain from chronic joint disorders. Aust J Physiother 2003;49(2):107-16.

[4] Rochkind S, Geuna S, Shainberg A. Chapter 25: Phototherapy in peripheral nerve injury: effects on muscle preservation and nerve regeneration. Int Rev Neurobiol 2009;87: 445-64.

[5] Lapchak PA. Taking a light approach to treating acute ischemic stroke patients: transcranial near-infrared laser therapy translational science. Ann Med 2010;42(8): 576-86.

[6] Maiman TH. Stimulated optical radiation in ruby. Nature 1960;187:493-4.

[7] Tunér J, Hode L. It's all in the parameters: a critical analysis of some well-known negative studies on low-level laser therapy. J Clin Laser Med Surg 1998;16(5):245-8.

[8] Jenkins PA, Carroll JD. How to report low-level laser therapy (LLLT)/photomedicine dose and beam parameters in clinical and laboratory studies. Photomed Laser Surg 2011;29(12):785-7.

[9] Martin LJ. Biology of mitochondria in neurodegenerative diseases. Prog Mol Biol Transl Sci 2012;107:355-415.

[10] Sarti P, Arese M, Forte E, Giuffrè A, Mastronicola D. Mitochondria and nitric oxide: chemistry and pathophysiology. Adv Exp Med Biol 2012;942:75-92.

[11] Antunes F, Boveris A, Cadenas E. On the mechanism and biology of cytochrome oxidase inhibition by nitric oxide. Proc Natl Acad Sci USA 2004;101(48): 16774-9.

[12] Lane N. Cell biology: power games. Nature 2006;443(7114): 901-3.
[13] Lubart R, Lavi R, Friedmann H, Rochkind S. Photochemistry and photobiology of light absorption by living cells. Photomed Laser Surg 2006;24(2):179-85.

[14] Huang YY, Chen AC, Carroll JD, Hamblin MR. Biphasic dose response in low level light therapy. Dose Response 2009;7(4):358-83.

[15] Huang YY, Sharma SK, Carroll J, Hamblin MR. Biphasic dose response in low level light therapy - an update. Dose Response 2011;9(4):602-18.

[16] Hashmi JT, Huang YY, Sharma SK, Kurup DB, De Taboada L, Carroll JD, Hamblin MR. Effect of pulsing in low-level light therapy. Lasers Surg Med 2010;42(6):450-66.

[17] Wu X, Alberico SL, Moges H, De Taboada L, Tedford CE, Anders JJ. Pulsed light irradiation improves behavioral outcome in a rat model of chronic mild stress. Lasers Surg Med 2012;44(3):227-32.

[18] Ando T, Xuan W, Xu T, Dai T, Sharma SK, Kharkwal GB, Huang YY, Wu Q, Whalen MJ, Sato S, Obara M, Hamblin MR. Comparison of therapeutic effects between pulsed and continuous wave 810-nm wavelength laser irradiation for traumatic brain injury in mice. PLoS One 2011;6(10): e26212-20.

[19] Saracino S, Mozzati M, Martinasso G, Pol R, Canuto RA, Muzio G. Superpulsed laser irradiation increases osteoblast activity via modulation of bone morphogenetic factors. Lasers Surg Med 2009;41(4):298-304.

[20] Neřmark AI, Muzalevskaia NI. Low-intensity laser radiation in preoperative preparation of patients with benign prostatic hyperplasia. Urologiia 2000;(1):11-5.

[21] Hashmi JT, Huang YY, Osmani BZ, Sharma SK, Naeser MA, Hamblin MR. Role of low-level laser therapy in neurorehabilitation. PM R 2010;2(12 Suppl 2):S292-305.

[22] Lin F, Josephs SF, Alexandrescu DT, Ramos F, Bogin V, Gammill V, Dasanu CA, De Necochea-Campion R, Patel AN, Carrier E, Koos DR. Lasers, stem cells, and COPD. J Transl Med 2010;8:16.

[23] Hahm E, Kulhari S, Arany PR. Targeting the pain, inflammation and immune response (PII) axis: plausible rationale for LLLT. Photon Lasers Med 2012;1(4):241-54. 
[24] Vatansever F, Hamblin MR. Far infrared radiation (FIR): Its biological effects and medical applications. Photon Lasers Med 2012;1(4):255-66.

[25] Ferraresi C, Hamblin MR, Parizotto NA. Low-level laser (light) therapy (LLLT) on muscle tissue: performance, fatigue and repair benefited by the power of light. Photon Lasers Med 2012;1(4):267-86.

[26] Vatansever F, Rodrigues NC, Assis LL, Peviani SS, Durigan JL, Moreira FMA, Hamblin MR, Parizotto NA. Low intensity laser therapy accelerates muscle regeneration in aged rats. Photon Lasers Med 2012;1(4):287-97.

[27] Marquina N, Dumoulin-White R, Mandel A, Lilge L. Laser therapy applications for osteoarthritis and chronic joint pain - A randomized placebo-controlled clinical trial. Photon Lasers Med 2012;1(4):299-307.

[28] Almuslet NA, Osman AE. Investigation of the effect of low power laser used in photodynamic therapy for treatment of psoriasis. Photon Lasers Med 2012;1(4):309-17.

\section{Arkady Mandel ${ }^{\star}$}

Michael R. Hamblin*

*Corresponding authors: Arkady Mandel, Theralase Inc., 102-29 Gervais Dr., Toronto, Ontario M3C 1Y9, Canada,

e-mail: amandel@theralase.com;

Michael R. Hamblin, Wellman Center for Photomedicine, Massachusetts General Hospital, Boston, MA, USA; Department of Dermatology, Harvard Medical School, Boston, MA, USA; Harvard-MIT Division of Health Sciences and Technology, Cambridge, MA, USA, e-mail: hamblin@helix.mgh.harvard.edu
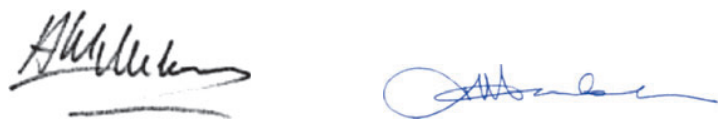

Arkady Mandel
Michael R. Hamblin 